\title{
Fine ways to fail to secure local realism
}

\author{
Soazig Le Bihan ${ }^{1}$ \\ University of Montana, Missoula, MT
}

\begin{abstract}
For more than twenty five years, Fine has been challenging the traditional interpretation of the violations of Bell inequalities (BI) by experiment. A natural interpretation of Fine's theorem is that it provides us with an alternative set of assumptions on which to put the blame for the failure of the BI, and a new interpretation of the violation of the BI by experiment should follow. This is not, however, how Fine interprets his theorem. Indeed, Fine claims that his result undermines other interpretations, including the traditional interpretation in terms of local realism. The aim of this paper is to understand and to assess Fine's claims. We distinguish three different strategies that Fine uses in order to support his interpretation of his result. We show that none of these strategies is successful. Fine fails to prove that local realism is not at stake in the violation of the BI by quantum phenomena.
\end{abstract}

Key words: A. Fine, quantum mechanics, locality, Local Realism, probabilities, Prism Models

Email address: soazig. lebihan@normalesup.org (Soazig Le Bihan).

URL: www. soaziglebihan.org (Soazig Le Bihan).

1 I wish to express my gratitude Guido Bacciagaluppi and Armond Duwell for insightful comments on earlier drafts of this paper. I also wish to thank an anonymous referee for helpful clarifications. 


\section{Bell-type theorems, Fine's theorem, and their interpretations}

\subsection{Bell-type theorems and experiments}

Bell-type theorems ${ }^{2}$ come in various versions, but most of them follow the following general scheme: from a set of assumptions $S$ are derived some inequalities (the Bell inequalities - henceforth the BI), which in turn are shown to be violated by some quantum statistical predictions. The conclusion follows by a simple modus tollens: no model of $S$-type, i.e. no model which satisfies all the assumptions in $S$, can recover all the statistical predictions of quantum mechanics.

Some experiments can be conducted which arguably correspond to testing the BI. ${ }^{3}$ A Bell-type experiment generally involves a measurement set up with two separated parts, or "wings", $A$ and $B$, and a source. At the source, a system is produced which is traditionally understood as constituted by two parts which travel in opposite directions corresponding to the two wings. On each wing, a measurement device (detector plus analyzer) which can be set to measure one

2 The original proof of Bell's Theorem, within a deterministic context, is in [5]. The result has been generalized to the stochastic case by Bell in [7] and by others, including [12],[13], [2], and [37]. The literature on Bell-type theorems is plentiful. Bell's papers are indispensable references: [5-8], all reprinted in [9], with some others in [10]. For a recent synthesis on Bell theorem, see [43].

3 The experiment performed in 1982 by Alain Aspect and his team in Orsay is considered the classic ([4], [3]). Redhead provides a table of the experimental results concerning the violations of the Bell Inequalities up to the last eighties in [39, p.108]. Many others have been conducted since, among which the ones by Zeilinger's team in Vienna and Gisin's team in Geneva are well known (see for example [55], [56]). 
of several observables on each side, labeled respectively $A_{1}, \ldots, A_{n}$ and $B_{1}$, $\ldots, B_{n}$, with the $A_{i}, B_{j}$ commuting for all $(i, j)$ and the $A_{i}, A_{j}$ and $B_{i}, B_{j}$ non commuting for every $i \neq j$. One then considers the single and double outcome distributions $P\left(A_{i}\right), P\left(B_{j}\right)$, and $P\left(A_{i}, B_{j}\right)$, usually for $i, j \in\{1,2,3\}$. The experiments to date are almost uncontroversially taken to be in agreement with the predictions of quantum mechanics. ${ }^{4}$ The upshot is thus that no model of the $S$-type can give an account of all quantum phenomena.

The interpretation of the experimental violation of Bell-type inequalities varies depending on the set of assumptions $S$ considered in the derivation. In most cases, $S$ is supposed to capture the idea of "Local Realism". The phrase "Local Realism" is widely used in the literature, even if there is no clear, uncontroversial definition of it. ${ }^{5}$ Roughly, a local realist model is characterized by the two following conditions:

Realism: measurement outcomes are determined before the actual measurement process;

Locality: measurement outcomes are locally determined; namely, the results of a measurement are independent of what is happening on remote systems.

Let us make these conditions more clear.

To provide quantum phenomena with a realist model in the above sense is

$\overline{4}$ There remains some debate on the so-called "detection loophole". For an analysis of the detection loophole, see the work of J.A. Larsson, for instance [35]. We'll come back to the problem of detection when dealing with Fine's prism models in Section 3.

5 See for example [32, p.61-2] for a "deliberately vague" characterization of local realism. 
the aim of hidden variable (h.v.) theories. By contrast to the orthodox interpretation of standard quantum mechanics, ${ }^{6}$ h.v. theories take it that one can supplement the quantum state with extra variables, ${ }^{7}$ such that the complete state of the system, determines the outcome of a given experiment. That said, the sense in which the outcomes are "determined" comes in various ways.

Arguably the strongest sense for such a determination is value definiteness. Value definiteness is the requirement that state of a system assigns definite values for all quantum observables, irrespective of whether a measurement is performed. If these values further encode determinate measurement results, h.v. models satisfying value definiteness are non-contextual, i.e. the measurement results do not depend on the measurement context. ${ }^{8}$ It is not necessary for h.v models to satisfy value definiteness in order to count as realist in the sense above.

It is not necessary that the outcomes be determined deterministically either. A physical transition between the times $t$ and $t+\delta t$ is said to be deterministic if and only if the state of a closed system submitted to this transition is

$\overline{6}$ It is well known that the quantum state, given as in standard quantum mechanics, does not allow one to predict definite outcomes for a given experiment: it only gives a probability distribution over a spectrum of possible outcomes. The orthodox interpretation takes it that no more complete specification of the physical situation can be made.

7 These are called "hidden" variables because we do not have direct access to their values, nor do we have control of them in practice

8 More will be said about contextual h.v. models in Section 3. For distinctions between types of contextuality, see for example [43, p.2], [39, p.135, 139-142] and $[14]$. 
completely determined at a time $t+\delta t$, given its state at $t .{ }^{9}$ Accordingly, a deterministic h.v. model is such that the outcomes of a given measurement are completely determined by the state of the system before measurement, possibly together with the measurement context. By contrast, a stochastic mode includes a stochastic dynamics at the "hidden" level, such that the complete state of both the system and the experimental context does not determine completely the result of the measurement. H.v. models can be stochastic.

We can now introduce the weakest interpretation of the notion of realism above, i.e. determinateness. Determinateness is satisfied if and only if each experiment will have definite results, which may be fixed deterministically or only stochastically by the state of the system, possibly together with the state of the experimental context, before measurement. It should be clear determinateness does not involve either determinism or (non-contextual) value definiteness.

Let us turn to the second aspect of Local Realism, i.e. locality. The notion of locality within Bell-type theorems and Bell-type experiments have been a central matter of controversy. Most commonly, locality is characterized in a deliberately vague way in terms of the prohibition of "influences" between spatially separated regions. ${ }^{10}$ The condition is traditionally supposed to be

$\overline{9}$ For a detailed study of the notion of determinism in the context of Bell experiments, in particular for a distinction between two-time determinism and dynamical determinism, see [16].

${ }^{10}$ Clearly, the notion of influence is vague, in particular when one wants to draw consequences concerning the relationships between the notion of locality in the Bell context and in Relativity Theory. This problem cannot be addressed in any details here for it would take us off course. For an extended study on this topic, see [36]. 
captured by a formal condition on probabilistic distributions over outcomeevents. ${ }^{11}$ "Locality" is then satisfied when the outcome-events $a$ and $b$ are statistically independent, each conditional on the complete specification of the states and measurement protocol at its respective end of the experiment. This is the famous factorization condition, or factorizabiliy:

$$
p_{\lambda}(a, b \mid i, j)=p_{\lambda}(a \mid i) p_{\lambda}(b \mid j)
$$

where $\lambda$ stand for the "hidden complete state" $12, i$ for the setting of the apparatus $A, j$ for the setting of the apparatus $B$. Whether or not factorizability is the correct way to formalize the requirement of locality has been the center of discussions over the past 30 years. The most common view takes factorizability as including a requirement of locality. ${ }^{13}$

In the end, the received view takes it that the experimental violation of the BI forces us to admit that no local (i.e. factorizable) realist (i.e. determinate) model can recover all quantum phenomena. This is the view which Fine has been challenging for more than twenty five years.

\footnotetext{
$\overline{11}$ This condition was introduced by Bell in [7, p. 37].

${ }^{12}$ Whether $\lambda$ is a single variable or a set of variables does not matter in the context of the following.

${ }^{13}$ Jarrett and Shimony (see [31] and [42]) showed independently (after Suppes and Zanotti in [44]), that factorizability divides up into two distinct conditions on the probability distributions of the outcome-events: Outcome and Parameter Independence. Parameter Independence is usually taken to be a locality condition, while Outcome Independence is interpreted in various ways, including in terms of separability and holism (see [30]). Another important interpretation of the Bell-type experiments is in terms of backward causation (see [38] and [17] for example).
} 


\subsection{Fine's theorem and its possible interpretations}

Fine proved the following theorem: ${ }^{14}$

Theorem 1 The following conditions on the correlations of a Bell-type experiment with $i, j \in\{1,2\}$ are equivalent:

(1) The BI hold for the probability distributions of the experiment;

(2) There is a deterministic hidden variable model of the experiment returning the observed outcome distributions (singles and doubles);

(3) There is a factorisable model of the experiment returning the observed outcome distributions (singles and doubles);

(4) There is a joint distribution for all four observables of the experiment $P\left(A_{1}, B_{1}, A_{2}, B_{2}\right)$, compatible with the observed outcome distributions (singles and doubles) as marginals;

(5) There are well-defined joint distributions for all pairs and triples of commuting and noncommuting observables compatible with the observed outcome distributions (singles and doubles) as marginals.

The result is multifold but two striking features deserve discussion. First, Fine's theorem provides us with alternative derivations of the BI: he shows that it is sufficient, for a probabilistic model to satisfy the BI, to have well defined joint probability distributions for all pairs and triples of observables, or to have well defined joint distributions for all four observables, whether these observables are compatible or not - let us call these PJPD, for Prob-

$\overline{14}$ The original proof is to be found in [21]; [22] is more philosophically concerned, and more ambitious in terms of the consequences Fine wants to draw from his result, and $[25]$ is making the point in a non-technical way. 
lematic Joint Probability Distributions. (We say"Problematic" because they cannot be correct because of the violation of the BI by experiment). Second, Fine's theorem establishes the converse of the original Bell derivation of the inequalities. ${ }^{15}$

A natural interpretation of Fine's result is as follows. An alternative derivation of the BI is provided: the BI hold in any probabilistic model in which the PJPD are well defined. Alternative assumptions are uncovered on which we can put the blame in a Bell-type modus tollens argument. No model in which the PJPD are well defined can give an account of all quantum phenomena. The ontological significance of this fact can be the subject of philosophical inquiry.

This is not the way in which Fine interprets his theorem. From 1982 on, he has been holding that his theorem does more than provide an alternative derivation from which additional interpretations of the ontological situation underlying Bell-type experiments can be made. Rather, Fine contends that the interpretation in terms of well defined joint probability distributions su-

$\overline{15}$ That is to say, Fine shows that any model satisfying the BI is equivalent to a deterministic h.v. model of the experiment. It should be noted that Fine's theorem also contains results about factorizable, and hence potentially stochastic, models. It shows that any factorizable model of the experiment is equivalent to a deterministic one. In fact, this is a slightly weaker result than the one Suppes and Zanotti obtained in [44], that any factorizable model of the experiment (in which one can have perfect anticorrelations) is in fact deterministic (see also [52] and [16, p.61-62]). This means that there is no genuinely stochastic factorizable model of the experiment. For this reason, this paper will focus on Condition (2) instead of (3) in the theorem when discussing the significance of Fine's theorem. That said, no result of this paper hinges on the assumption of determinism. 
persedes other interpretations of the violation of the BI. In particular, the traditional interpretation in terms of local realism would be threatened. Consider the three following quotations, two from the original papers, the other from a more recent one:

Our investigations suggest that what the different hidden variables programs have in common, and the common source of their difficulties, is the provision of joint distributions in those cases where quantum mechanics denies them. ${ }^{16}$

Finally, I believe that Proposition (1) - conjoined with the other two [items 2, 3, and 5 above]- shows what hidden variables and the Bell inequalities are all about; namely, imposing requirements to make well defined precisely those probability distributions for non commuting observables whose rejection is the very essence of quantum mechanics. ${ }^{17}$

The Bell inequalities have a purely probabilistic content $[\ldots] .{ }^{18}$

As it stands, such claim does not seem to hold. It is a simple matter of logic that the theorem does not undermine the other derivations, nor conclusions drawn from them. Further argumentation is needed to support Fine's strong interpretation of his results.

\footnotetext{
$\overline{16}[22, \mathrm{p} .1309]$

$17[21$, p.294]

$18[28, \mathrm{p} .3]$
} 


\subsection{Fine's three lines of argumentation}

In this paper, we distinguish between, and object to, three lines of arguments that Fine uses to defend his strong interpretation.

Section 2 deals with Fine's first line of argument. Fine claims that his theorem uncovers an additional assumption in the traditional derivation which is sufficient to have the BI satisfied. If true, then the violation of the BI is not necessarily to be interpreted in terms of Local Realism. However, as we shall point out, Fine's argument only holds for non-contextual h.v. models, i.e non-contextual. For contextual h.v. research programs, the interpretation in terms of local realism is highly relevant: indeed, it proves that they must be non-local to be compatible with all quantum statistical predictions.

Section 3 focuses on a second line of argument, which is based on the existence of the so-called Prism Models. Prism Models are local realist models which reproduce the violation of the BI. Fine seems to conclude from this that local realism is not at stake in the violation of the BI. Against this argument, we shall point out that Prism Models are not models of quantum probabilities, but only of the actual experiments. Contra quantum theory, Prism models deny that any systems can be detected in any experimental configuration. Hence, their existence does not threaten the traditional view that no local realist classical probabilistic model can return all statistical predictions of quantum mechanics.

In Section 4, we turn to Fine's last line of argumentation, which is based on the converse of his new derivation of the BI: that the BI's holding implies the PJPD to be well defined. From this, Fine wants to deduce that h.v. models 
for quantum probabilities start right from the beginning with an inconsistent set of hypotheses. The argument is not all clear, but can be reconstructed in the following way: ${ }^{19}$

(1) In accepting QM, the h.v. investigator is committed to rejecting the existence of joint probabilities for incompatible observables;

(2) By the converse of the new derivation, anyone committing to a h.v. model from which the $\mathrm{BI}$ are derivable is also committed to the existence of joint probabilities for incompatible observables.

Svetlichny et alii have shown that (2) is not well supported. We shall recall their argument, and proceed to criticize (1), in pointing out that, contrary to what Fine assumes, the acceptance of a theory does not include a specific interpretation of probabilities. Applied to (1), this means that, in accepting quantum mechanics, one is not committed to anything like "the existence" or the "non-existence" of well defined probabilities in the sense needed by Fine.

\section{A hidden assumption in the traditional derivation?}

One way to construct hidden variable models is to construe observables as random variables defined over a common classical probability space. Such construction is usually referred to as an "ensemble representation". It is a formal consequence of an ensemble representation that the joint distributions are well-defined for all pairs of observables, commuting or not. Further, these joint distributions are compatible with the singles as marginals. Hence, by

$\overline{19}$ This is inspired by the work of Svetlichny, Redhead, Brown and Butterfield in $[45]$. 
Fine's theorem, some BI hold for such models. Fine holds that this supports the claim that his theorem uncovers a hidden assumption in the traditional probabilistic models, an assumption which alone is sufficient to make the BI to hold:

[...] any ensemble representation must make well defined joint distributions for incompatible observables (like position and linear momentum, or spin in skew directions), for the function associated with these observables will be random variables over a common space and these always have well-defined joints. $^{20}$

In this section, we shall first explain how ensemble representations indeed include the definition of the PJPD. We shall then proceed to criticize Fine's strong conclusion in pointing out that a large class of hidden variable theories do not construe quantum observables as random variables over a common classical probability space. For this class of h.v. theories, Fine's argument does not stand.

\subsection{Ensemble representations and joint probabilities}

To construct an ensemble representation is a way to reduce statistical distributions of outcomes to determinate but unknown states. In our case, let the "complete state" of a system be the quantum state plus the set of hidden variables $\lambda$ which together determine the results of measurements. Consider now the set $\Lambda$ of all possible hidden variables $\lambda$. Even if all we know about a system is confined to the probability distribution over a spectrum of possible

$\overline{20[24, \mathrm{p} .45]}$ 
outcomes given by $|\psi\rangle$, we want the system under consideration to be really in one of the complete states corresponding to some variables $\lambda$ (or the state $|\psi\rangle$ really describes an ensemble of systems, each of which is in one of the complete states corresponding to the $\lambda$ ). For each $|\psi\rangle$, this set $\Lambda$ is then structured as a classical probability space, that is to say, is equipped with a probability measure on its Borel sets $\mathcal{B}_{\Lambda}$. Thus, for a given $|\psi\rangle$, there is a probability density $\rho^{|\psi\rangle}(\lambda)$ of the possible hidden parameters $\lambda$ over the space $\Lambda$. That is to say,

$$
P^{|\psi\rangle}(I)=\rho^{|\psi\rangle}(\lambda) d \lambda
$$

represents the probability that the hidden variables lie in the interval $I=$ $[\lambda, \lambda+d \lambda]$

What remains to be done is to provide a formal way of connecting the statistical distributions of outcomes with this probability measure. To this aim, every observable $Q_{i}, i \in\{1, \ldots, n\}$, is defined as a random variable on the common probability space $\Lambda$, that is, every $Q_{i}$ is represented by a real-valued function $\left[Q_{i}\right]$ defined over $\Lambda$. For a given state, any observable has a range of determinate values associated with the possible $\lambda$. A consequence of construing observables as random variables over $\Lambda$ is that the probability structure $\left(\Lambda, \mathcal{B}_{\Lambda}, \rho^{|\psi\rangle}(\lambda)\right)$ induces one on the Borel sets $\mathcal{B}_{E_{i}}$ of the ranges $E_{i}$ of the functions $\left[Q_{i}\right]$ (in general the $E_{i}$ are some subset of the real numbers). A new probability measure is thus defined on the subsets $F$ of the $\mathcal{B}_{E_{i}}$, that is, the probability $P_{\left[Q_{i}\right]}^{|\psi\rangle}(F)$ that the function $\left[Q_{i}\right]$ of the observable $Q_{i}$ takes its value in $F$. It is defined in the following way: 


$$
\forall Q_{i}, \forall F \subset \mathcal{B}_{E_{i}}, P_{\left[Q_{i}\right]}^{|\psi\rangle}(F)=P^{|\psi\rangle}\left[\left(\left[Q_{i}\right]\right)^{-1}(F)\right]
$$

So, the probability structure on the set of hidden variables is projected on the space of the possible values of each observable. Thus, the statistical distributions of outcomes given by $|\psi\rangle$ for a given observable are reduced to statistical distributions of hidden variables.

Further, since the functions $\left[Q_{i}\right]$ are defined as random variables on a common probability space, any Borel function $f\left(\left[Q_{1}\right], \ldots,\left[Q_{n}\right]\right)$ is in turn a random variable on the Cartesian product $\Lambda^{n}$, and there is a new probability measure defined on the Borel sets of its range, fully characterized by $\rho^{|\psi\rangle}(\lambda)$ on $\mathcal{B}_{\Lambda}$. In particular, for all pairs of observables $\left(Q_{1}, Q_{2}\right)$, whether compatible or not according to quantum formalism, the joint probability $P_{\left[Q_{1}\right],\left[Q_{2}\right]}^{|\psi\rangle}$ and the random variable $f\left(\left[Q_{1}\right],\left[Q_{2}\right]\right)$ is defined for all Borel function $f$ such that they satisfy, for $F_{1}, F_{2}$, Borel subsets of respectively $\mathcal{B}_{E_{1}}$ and $\mathcal{B}_{E_{2}}$.

$$
P^{|\psi\rangle}\left[\left(\left[Q_{1}\right]\right)^{-1}\left(F_{1}\right) \cap\left(\left[Q_{2}\right]\right)^{-1}\left(F_{2}\right)\right]=P_{\left[Q_{1}\right],\left[Q_{2}\right]}^{|\psi\rangle}\left(F_{1} \times F_{2}\right)
$$

In other words, the probability that two observables take jointly some given values is fully determined by considering the intersection of the sets of hidden variables respectively corresponding to the given values.

Finally, the quantum expectation values are recovered as the expectation values of the functions corresponding to the observables along the the lines of the usual probability rules. That is, given a certain state, a simple integration over the whole range $\Lambda$ of the possible $\lambda$ provides the expectation value of any of the observables or of any Borel function $f\left(\left[Q_{1}\right], \ldots,\left[Q_{n}\right]\right)$ of the observables. 


$$
<f\left(\left[Q_{1}\right], \ldots,\left[Q_{n}\right]\right)>^{|\psi\rangle}=\int_{\Lambda} f\left(\left[Q_{1}\right], \ldots,\left[Q_{n}\right]\right)(\lambda) . \rho^{|\psi\rangle}(\lambda) . d \lambda
$$

To summarize, such h.v. models amount to constructing an ensemble representation for all the observables, which includes the definition of joint probabilities for all observables returning the singles as marginals, whether these observables are commuting or not. By Fine's theorem, the Bell inequalities hold for any probabilistic model of this kind.

Granted then, it is the case that any probabilistic model in which the observables are construed as random variables over a common classical probabilistic space entails that the PJPD are well defined. For these models, whatever the set of other assumptions they may include, whether it be locality, realism or anything else, the PJPD being well defined alone can be blamed for their violating the BI.

This alone, however, does not imply that Fine's theorem undermines the interpretation of Bell-type results in terms of local realism. For it is not the case that all hidden variable probabilistic models construe observables as random variables over a common classical probabilistic space. In particular, Fine never considers contextual h.v. research programs.

\subsection{Hidden variable models outside the scope of Fine's argument}

In arguing that his theorem shows that the traditional derivations of the BI include a hidden assumption about the definition of the PJPD, Fine seems to pursue two different aims. One aim is to dismiss h.v theories as a whole in

saying that "what the different hidden variables programs have in common, 
and the common source of their difficulties" ${ }^{21}$ is precisely the definition of the PJPD. Thus, h.v. research programs, whether local realist or not, would simply be dead ends. A second aim is to deflate the significance of Bell-type results: the PJPD being well defined would be what the BI are all about. In particular, the violation of the BI by experiment would not mean that h.v. theories have to be non-local in order to be compatible with all quantum phenomena.

Fine's argument presented above fails to help to achieve any of these aims. This is because the classical construal of the observables as random variables over a common probability space, which Fine shows to include the assumption of well defined joint probabilities, is non-contextual. But, first, we did not need to wait for Fine's theorem to know that no non-contextual h.v. theory can model quantum probabilities. Second, there are contextual h.v. theories, which do not include the definition of the PJPD, and thus, which fall outside Fine's argument. For such models, the derivation in terms of local realism remains highly relevant, for it forbids them to be local. Let us make these points in more detail ${ }^{22}$.

Hidden variable theories are said to be contextual, if and only if the value of the observable obtained by any measurement process is not fully determined by the complete state of the system, but depends on what other quantities are simultaneously measured, or of the state of the measuring apparatus or any other details of the measurement context. Shimony 1984 made a distinction between algebraic and environmental contextuality. Algebraic contextuality is when the value of a quantity measured depends on other quantities measured.

\footnotetext{
$\overline{21}[21$, p. 1309$]$

${ }^{22}$ For similar points, see Shimony in [41].
} 
Environmental contextuality is when the value of a quantity depends not on other quantities measured but on other features of the measurement context.

The idea of contextuality is more familiar than it seems. Let us take a rather simple analogy. Consider a horse race. Whether Quick Silver, your favorite horse, is going to win the race or not may depend on various factors. One factor could be the degree of humidity of the ground: for example, Quick Silver, even though an excellent runner on a dry ground, might be uncomfortable on a muddy soil. Whether Quicksilver is going to win presumably depends on which other horses are running the race as well. If so, the outcome of the raceexperiment depends on both the environmental context (the state of the track) and on the algebraic context of the race (What other horses are "measured"). It is both environmental and algebraic contextual.

Now, that there can be no non-contextual h.v. model compatible with all statistical predictions of quantum mechanics, was famously shown by Gleason in his profound theorem in 1957 for Hilbert space of dimension greater that two, ${ }^{23}$ and in a simplified version of the theory by Bell in 1966, ${ }^{24}$ as well as by Kochen and Specker 1967 theorem $^{25}$. Hence, Fine's theorem does not provide anything new as far as non-contextual h.v. theories are concerned. ${ }^{26}$

Further, Fine cannot hope to dismiss all h.v. research programs on the basis that all non-contextual h.v. theories include the definition of the PJPD. The entire class of contextual h.v. theories simply falls outside Fine's argument. Bohm's theory is such a contextual h.v. theory, which returns all statistical

\footnotetext{
$\overline{23 \text { in }[29] .}$

24 in $[6]$.

25 in [33].

${ }^{26}$ See Shimony in [41] for a discussion of the case of dimension 2.
} 
predictions of quantum mechanics within the bounds of current empirical accuracy. It does not include the definition of the PJPD, and thus does not necessarily imply that the BI hold by Fine's theorem. This is because Bohm's theory does not use the classical construal of observables as random variables over a common probability space, but the framework of statistical variables.

A statistical variable is real-valued function with a probability measure directly defined on the Borel subsets of its range. This can be contrasted with random variables which we considered up to now. A random variable is a real valued function defined on the space of complete states, or on some subset of the space of complete states. In the case of a random variable, there is a probability measure on the possible values of the variable (which derives from the probability measure on the states). Therefore, a random variable is a statistical variable but the converse does not hold, as the example of Bohm's theory shows.

\section{POSSIBLE CUT: FOLLOWING PAR.}

Within Bohm's theory, the result of a given measurement is encoded in the position of the "pointer", for which there is always a well defined probability distribution. While of course the result of a given measurement depends on the $\lambda$, which values of the $\lambda$ are underlying which positions of the pointer depends on the context of measurement. Thus, even if the outcomes are construed as deterministically arising from an underlying physical situation, the observables are not construed as random variables on the common space $\Lambda$. Instead, the various experimental set-ups correspond to statistical variables.

Now, given two statistical variables, the joint distribution is generally not defined. By contrast, two random variables, as explained above, whenever 
they are defined on a common domain, have their joint distributions well defined. Clearly then, by Fine's theorem, the BI do not necessary hold in a statistical variables model of the quantum distributions. Any h.v. models which uses statistical variables instead of random variables for construing the hidden variable falls outside of Fine's argument.

\section{POSSIBLE CUT: DISCUSSION OF MINIMAL REALISM and BOHM}

It is worth noting that Fine has been advocating the framework of statistical variables as the appropriate framework for quantum probabilities since (at least) 1968, precisely because it allows for the PJPD not to be necessarily well defined. ${ }^{27}$ Fine claims that statistical variable models admits a "natural" interpretation he dubs "Minimal Realism", according to which the following objects and properties are real: ${ }^{28}$

(1) physical objects - corresponding to the theoretical systems;

(2) generic features which can take different forms - corresponding to the observables;

(3) particular forms of the generic features - corresponding to the values of the observables;

(4) distributions of probability on the spectrum of particular forms of the generic features - corresponding to the distributions of probability on the spectrum of values of the observables, ascribed by the quantum state.

Thus, the theoretical state of a quantum system is associated with an objective physical object (say, a quantum coin), an objective property (say, Quantum Face), a spectrum of objective forms of the property (Quantum Head and

$\overline{{ }^{27} \text { See }}$ [18], [19], [20], and [24, chap. 4].

${ }^{28}$ See [24, p.160 sq.]. 
Quantum Tail), and the objective probability measure over this spectrum of forms (an even distribution for instance between Q-Heads and Q-Tails if our quantum coin is fair).

In minimal realism, probability distributions are non-reducible, objective features of the world: they are second order properties (properties of properties) of the object. Each property possesses as a property the probability distribution over its different forms. These distributions determine the outcome of an experiment. Thus, the framework of statistical variables, when interpreted with Minimal realism, can be seen as a non-deterministic but determinate model for quantum probabilities.

Not all h.v. models using the framework of statistical variables have to take the probabilities as fundamental though. Determinism is still an option. For example, Bohm's theory uses the framework of statistical variables, but maintains an ignorance interpretation of probabilities. Within Bohm's theory, quantities are interpreted either as categorical properties (for the quantities with continuous spectra such as position) or as dispositions (for all others). The dispositional properties considered by Bohm's theory can be seen as being reducible. ${ }^{29}$. Reducible dispositions are not ontologically significant: they reduce to categorical properties together with the experimental context. On Bohm's theory, measurement outcomes are determined by the underlying situation, including the initial positions of the particles, the initial wave-function, and the experimental context. The probability distribution associated with any given measurement arises from our ignorance of the underlying situation, in particular from our ignorance of the initial configuration of the particles.

\footnotetext{
${ }^{29}$ See Clifton and Pagonis on this in [14].
} 
So, in contextual h.v. theory (whether deterministic or not), the PJPD are not necessarily well-defined. Given that Bell has shown that the BI are derivable from local realism in a contextual setting, ${ }^{30}$ the violation of the BI by experiment is still relevant for such theories. Indeed, it implies that, since they endorse the assumption of "realism", contextual h.v. theories have to be non-local in order to be compatible with all statistical predictions of quantum mechanics. Bohm's theory is one such non-local contextual h.v. theory.

Thus, Fine's first line of argument can deflate neither the importance of h.v. research programs nor the significance of Bell-type results for these programs.

\section{A de facto argument: Fine's Prism Models}

Fine has constructed local realistic models for the Bell experiments, the socalled Prism Models. ${ }^{31}$. Fine seems to believe that the existence of his Prism Models implies that the traditional interpretation of the violation of the BI, according to which no local determinate h.v. theory can recover all quantum statistics, is threatened:

At this stage, however, one thing seems clear, and not acknowledged well in the recent literature. It is that Bell-type arguments and the experiments which support them cannot be straightforwardly understood as arguments against, or experimental refutations of, locality. ([23, p. 293])

\footnotetext{
$\overline{30}$ in $[6]$. $[48],[47])$
}

31 The original work is in [23], followed among other by [24, chap. 4], [26], and [27]. Others have gotten interested in Fine's models, like Larsson ([34]) and Szabó ([46], 
To the extent to which [to construct prism models] can be accomplished it undermines the experimental argument against local hidden variables, ... . $([26$, p. $475-476])$

In order to draw such conclusions, Fine needs to argue that (1) Prism Models are deterministic, local realistic models, which return the outcome distributions of actual Bell-type experiments; (2) Prism Models provide a probabilistic model of the quantum probabilities; Hence (3): the traditional interpretation of Bell-type results in terms of local realism is threatened.

In the following, we present the construction of Prism Models, thus granting (1), but then refute (2), thus showing that (3) does not follow.

\subsection{Prism Models}

Prism Models are probabilistic models characterized by the feature that observables do not in general have well defined probability distributions on all complete states $\lambda \in \Lambda$. More precisely, there are some quantum states, for which there are some hidden variables, for which there are some observables, on the ranges of which no probability distribution is defined. From a mathematical point of view, the idea is just that, while the observables $Q_{i}$ are separately construed as random variables, they are defined on different subsets $\sigma\left(Q_{i}\right)$ of $\Lambda$, thus being associated with partial real-valued functions on $\Lambda$.

Note that this is different from saying that some observables have a zero probability associated with some range of outcome for a given state. or that, in other words, a given state has probability zero to display such or such outcome 
associated with certain observables or experimental configurations. Instead, in the framework of partial random variables, no probability distribution is defined for these states which fall outside the domain of the function of the observable considered.

Now, another observable $Q_{j}$ will not in general have its function $\left[Q_{j}\right]$ defined on the $\lambda \in \sigma\left(Q_{i}\right)$. In other words, $\sigma\left(Q_{i}\right)$ in general includes states in and outside the domain of the function $\left[Q_{j}\right]$ associated with the measurement of $Q_{j}$. If we happen to measure $Q_{i}$ and $Q_{j}$ at the same time, the set of hidden states for the joint systems on which the joint measurement is defined is the intersection $\sigma\left(Q_{i}\right) \cap \sigma\left(Q_{j}\right)$. So, whether you measure $Q_{i}$ and $Q_{j}$ together or separately makes a difference as to which set of complete states is selected. In some cases, the intersection may be empty for some observables, so that some joint distributions may not be defined. Typically, in the case of a Bell-type experiment, the joint distribution over all observables is not defined, because the intersection of all domains of all the functions associated with all the observables is empty.

More needs to be said about the interpretation of Prism Models. That the observables are associated with partial real-valued functions on $\Lambda$ is interpreted in terms of defectiveness. For a given state $|\psi\rangle$, the $\lambda$ encodes for determinate values of the outcomes, but it also encodes for some physical, predetermined property (or properties), defectiveness, that makes the system under consideration suitable for certain measurements, and not for others. An $Q_{i}$-defective system just cannot respond to the experimental configuration (an analyzerdetector assembly) corresponding of the observable $Q_{i}$ and hence will not "show up" in the sense that it will not be counted by the detector. Applied to a Bell-type experiment, given the experimental configuration or observable 
$Q_{i}^{A}$ at the $A$ end of the experiment, $\lambda$ is in $\sigma\left(Q_{i}^{A}\right)$ if and only if an $A$-system of type $\lambda$ will be detected by any detector associated with a $Q_{i}^{A}$-analyzer.

Fine managed to show that there are Prism Models for the actual Bell experiments with two observables on each end. That is to say, it is possible to divide $\Lambda$ in subsets and define the four observables considered in the experiments over these subsets, such that the single and double probability distributions given by the model are consistent with the observed outcome distributions which violate the Bell inequalities.

In Prism Models, some underlying (even if possibly hidden) properties, associated with the variables $\lambda$, fully determine the outcomes of our experiments, including whether or not the system considered will be detected or not. So, Prism Models are determinate. Prism Models are even deterministic. Finally, Prism Models are local. Granted then, Fine has constructed local realistic models returning the statistical distributions of the actual experiments for dimension $2 \times 2$. Szabó has further proved the possibility of Prism Models for $n \times n$ dimensional systems. ${ }^{32}$ These are impressive results. However, they do support Fine's strong claim about the irrelevance of the notions of locality and realism for the interpretation of Bell-type experiments, as we shall now show.

\subsection{Prism Models, the experiments, and quantum mechanics}

Fine suggests that it is because the joint distributions are not defined that Prism Models can violate the Bell Inequalities in the right way. He claims $\overline{32 \text { in }[46]}$. 
that Prism Models keep the structural features of the probabilistic model which codify the "local realistic" character of the observables, while avoiding the assumption of the existence of the joint probabilities, which he deems responsible for the Bell inequalities to obtain. ${ }^{33}$ We argue in this subsection that this is not the case. The reason why Prism Models are successful is our actual failure of detection of all quantum systems. We will further argue that Fine's way to avoid that the PJPD makes Prism Models stand in contradiction with quantum theory. While providing an alternative model for the actual statistical results, Prism Models do not provide an alternative model for quantum probabilities. $^{34}$

Fine's Prism Models are successful because of a feature of the actual realizations of Bell-type experiments, namely the fact that not all systems that are produced at the source are detected by the measurement apparatuses. Our statistical measure of Bell-type correlations takes into account only these particles that are "coincidentally" detected by both detectors, not the original ensemble produced at the source. Prism models draw on this feature of the actual experiment to recover the probability distributions of the outcomes.

The usual way in which we interpret failure of detection is in terms of the

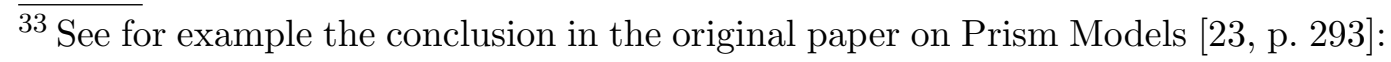
"... it is entirely possible that locality will survive this critical examination and that other principles - like the random variables framework - will go by the wayside instead.".

${ }^{34}$ What is striking is that Fine is well aware of the fact the Prism Models are only models of the actual experiment, not a general h.v. theory for quantum probabilities ([23, p. 280], [26, p. 475-476]). He fails to see, however, that this implies that his strong conclusion, as in the quotes at the beginning of this section, does not follow. 
deficiency of the detectors used. We further assume that such deficiency is random, and hence, that the sample of systems we actually detect is a fair one. This is called the "enhancement hypothesis". ${ }^{35}$ Fine proposes an alternative interpretation for failure of detection in the actual experiments. Within Prism Models, instead of being due to the random deficiency of our detectors, failure of detection reflects a physical property of the systems, i.e. defectiveness. Within the formalism of Prism Models, the property of defectiveness corresponds to the observables' being construed as partial random variable.

While giving room for a local deterministic account of the actual experimental results, the construal of the observables as partial functions on $\Lambda$ makes Prism Models stand in contradiction to quantum mechanics (as normally construed). If interpreted in terms of defectiveness, Prism Models predict that not all particles will be detected by a given experimental context. If not interpreted in terms of defectiveness, they still fail to make predictions (even probabilistic ones) for some runs of the experiments. In any case, Prism Models predict that they are some quantum systems $\left(Q_{i}\right.$-defective systems $)$ and some experimental context $\left(Q_{i}\right)$, for which no probability distribution over possible outcome-event is defined.

On the contrary, quantum mechanics predicts that any quantum system should respond to any given experimental question. Indeed, according to the usual quantum measurement theory, any quantum system can be appropriately coupled to some other system which will act as a perfect measuring apparatus. ${ }^{36}$ This motivates the usual interpretation according to which any deficiency in

\footnotetext{
$\overline{35}$ On this, again, see [35] and reference therein.

${ }^{36}$ We do not consider the limitations shown by Araki and Yanase in [1]. They can be disregarded for present purposes.
} 
the experiment is in one of our detectors - it is a technical problem - and does not reflect any property of the system being measured.

Because Prism Models contradict quantum mechanics, their existence does not support the strong claim held by Fine, that there are models consistent with all quantum statistical predictions in which local realism is secured. It also does not support the claim that locality and realism are not at stake in the violation of the BI.

That said, what it does support is the claim that there are local deterministic models consistent with the actual statistics of Bell-type phenomena. Now, that Prism Models are local deterministic models of the experiments to date, is, we believe, worth considering for further investigation. It would be certainly worth pursuing a research program to experimentally assess whether or not there is a detection efficiency limit. Szabó investigates to what extent Prism Models can be refuted by experiment on the basis of the detection efficiency rates. His conclusion is that actual experimental results still do not constitute a serious objection to Prism Models. Fine's models might be more relevant than they seem.

\section{POSSIBLE CUT: DISCUSSION OF DEFFECTIVENESS}

If further experimental investigation was favorable to Prism Models, some further interpretational work would still have to be done. The interpretation in terms of defectiveness seems unsatisfactory as it stands. What we have is that there are some complete states which possess some physical property such that some observables cannot be measured. Fine does not give any further interpretation of this property. At least three further interpretations are possible. First, if an observable corresponds to some determinate property-assignment, 
one interpretation is that the property corresponding to the observable is not measurable. Unless Fine could provide an explanation for why this happens, such an interpretation goes against one of the most fundamental assumptions in the methodology of experiment about the possibility of measurement. Moreover, it might run into problems with Kochen-Specker-type theorems. Another interpretation would be that the observable corresponds to a generic property, but the value of this property is unmeasurable because it is indefinite. The idea is that some properties do not have any determinate value, that could be correlated either deterministically or stochastically with the measurement context. This could correspond to the notions of "spread quantities" or "inexact values" that have been defended by some authors. ${ }^{37}$ However, Fine seems to reject this option himself: he accepts the objections leveled against such notion of spread quantities. ${ }^{38}$ Yet another interpretation is that the observable corresponds to a generic property, but there are some states that simply do not "have" this generic property, in any form, exact or spread. This obviously needs more fleshing out, but it might be what Fine has in mind, considering the concluding remark of [22]:

After all, if we hold that probabilities (including joint probabilities) are real properties, then some observable may simply not have them. ${ }^{39}$

That said, Fine never seriously investigates such issues. Indeed, Fine himself admits that Prism Models are somewhat too "cheap" and too "easy". 40 The $\overline{37}$ See for example Teller in [49], [50], and [51].

${ }^{38}$ See [24, p.161, note 16].

39 [22, p.1310]

${ }^{40}$ See $[24$, p. 56 sq.]. Fine formulates the same kind of qualifications as to the philosophical or physical significance for his local model in response to the Hardy theorem in [28]. 
aim of constructing these models was primarily negative, that is, it was to undermine the traditional interpretation in terms of local realism. This, however, as we have argued, is just not what Prism Models can be used for.

\section{Beyond the alternative? Fine's strong claim}

Fine's last line of argumentation in favor of his strong claim uses the converse of his derivation of the BI, that is, that any model in which the BI hold is equivalent to a model in which the PJPD are well-defined. From this, Fine wants to deduce that any set of assumptions from which the BI are derivable has, in Fine's terms, the "existence of well defined joint probabilities" as a consequence, including for non-commuting observables. Given that the definition of these joint probabilities is forbidden by quantum theory, h.v. programs start right from the beginning, Fine's argument goes, with an inconsistent set of hypotheses.

Fine's argument can be reconstructed as coming in two steps:

(1) In accepting QM, the local realist is committed to rejecting the existence of joint probabilities for incompatible observables;

(2) By the converse of the new derivation, anyone committing to a h.v. model from which the BI are derivable is also committed to the existence of joint probabilities for incompatible observables.

Svetlichny, Redhead, Brown and Butterfield have investigated this argument. ${ }^{41}$ They accept (1) but show that (2) is not well supported. In the following, we $\overline{41 \text { in }[45] .}$ 
shall briefly sum up their argument against (2) and then modestly contribute to undermining Fine's argument in raising some objections against (1).

Svetlichny et alii reject (2) on the basis that Fine's theorem does not show that the BI's holding implies the joint probabilities for incompatible to exist in the sense of being physically real. What has been shown instead is a weaker result, i.e. that the BI's holding implies the formal definition of the joint probabilities. But the local realist might be happy to accept the joint probabilities as being defined formally, but without giving them any physical significance.

Fine himself gives a criteria, (jd), ) for the joint probabilities to be considered as physically real. ${ }^{42}$.

(jd) - Observables $A_{1}, \ldots, A_{n}$ of a quantum system obey (jd) just in case, for every $n$-place Borel function $f$, there is an observable of the system with operator $f\left(A_{1}, \ldots, A_{n}\right)$, and for all states $\Psi$ of the system there is a probability measure $\mu_{\Psi, A_{1}, \ldots, A_{n}}$ on the Borel sets of $R^{n}$ that returns the quantum single distributions $P_{A_{i}}^{\Psi}$ as marginals and is such that for all borel sets $S$ :

$$
\mu_{\Psi, A_{1}, \ldots, A_{n}}\left(f^{-1}(S)\right)=P_{f\left(A_{1}, \ldots, A_{n}\right)}^{\Psi}(S)
$$

Note that (jd) is not what Fine shows to be equivalent to the BI's holding in his main theorem. In the theorem the definition of the PJPD depends on the state and on the four observables considered in the experiment. So, if (jd) is the appropriate formalization of the idea that the joint probabilities exist,

$\overline{42}$ The following is taken from [22]. Fine presents (jd) as a criteria for the joint probabilities to exist in $[20$, p. 31]. 
Fine's theorem cannot be used to legitimate (2). Further investigation about the relationships between (jd) and the BI's holding is needed.

Fine manages to show that violations of the BI are also violations of $(\mathrm{jd})$, but not the converse, which is needed for (2) to hold. Now, as Svetlichny et alii notice, the problem is that the converse is false. A simple way to see this is to appeal to another of Fine's results, i.e., that (jd) is equivalent to all the observables' pairwise commuting ${ }^{43}$. Now, there are many cases in which the BI are satisfied while not all observables commute. ${ }^{44}$ So, if (jd) is the appropriate formalization of the idea that the joint probabilities exist, then it is not the case that a commitment to the non-existence of the joint probabilities (due to a commitment to QM) implies commitment to the BI to be violated, and (2) is undermined.

One could object that (jd) is too strong, and that weakest assumptions (such as the ones appearing in Fine's main theorem) express the idea of the physical existence of the PJPD. Svetlichny et alii's study allows to counter such an objection. Indeed, they show that, if we look at the probabilities through the limiting relative frequencies generated on random sequences in the real world, there are some models of the Bell-type experiment which satisfy the BI while violating (jd). ${ }^{45}$ So, it seems that all what Fine's theorem proves is that the

\footnotetext{
$\overline{43}$ Theorem 7 in [22]

${ }^{44}$ Any experiment in which the observable measured on one side remains the same, while the observable on the other side varies: $A_{1}=A_{2}$ and $B_{1} \neq B_{2}$ will have the BI satisfied, while (jd) violated.

${ }^{45}$ See [45, Section 3, p. 395]. For other arguments against the idea that Fine's theorem shows more than the formal definition of joint probabilities, see [40, p. 123] and [11, p. 142-145].
} 
models satisfying the $\mathrm{BI}$ are equivalent to models in which the PJPD are formally defined but unlikely to have any physical significance.

So, (2) does not hold. Let us turn to (1), i.e. that in accepting QM, the local realist is committed to rejecting the existence of joint probabilities for incompatible observables. It seems to us that 1 . consists in at least three components. The first component is the assumption that h.v. programs are committed to accepting quantum theory. The second component is that to accept quantum theory is sufficient for being realist about quantum theory, in particular about quantum probabilities. This is to say that to accept the theory commits one to a certain interpretation of the probabilities involved in the theory. The final component is that to be realist about a theory that contains probabilities is sufficient for being committed to the "existence" of only the probabilities that are well defined within the theory. Our contention is that none of these components is trivial. Each can be avoided in hidden variable programs, so that Fine's argument does not stand.

First, not all hidden variable programs presuppose the quantum formalism. As Shimony systematically points out, there are different types of h.v. programs, with different sets of assumptions and tackling different issues. ${ }^{46}$ An important part of these programs aims at assessing whether some specific kind of probabilistic models are compatible with all statistical predictions of quantum mechanics. The construction of the probabilistic models generally does not presuppose the quantum formalism, quite the contrary. Famously, the original derivation of the BI by Bell does not presuppose the quantum formalism. The point of some of these research programs is to show what kinds

\footnotetext{
$\overline{{ }^{46} \text { See }[41]}$.
} 
of probabilistic models are not compatible with all statistical predictions of quantum mechanics. One can then investigate the significance of the failure of such models from an interpretational point of view: what the world could be like, since such and such probabilistic models are impossible? Such investigation guides other, more positive, research programs, which try out new theories which are empirically equivalent to standard quantum mechanics, up to our current level of empirical accuracy. Bohm's theory is obviously a case in point (although historically it developed in a more complicated manner).

Concerning the second component, it is not clear that Bell-type research programs are as committed to scientific realism as Fine wants them to be. To accept a theory can involve at least two different options:

(1) to believe that the theory is true or approximately so, or,

(2) to take the theory as empirically adequate.

One might want to take an empiricist stance towards quantum theory, but still investigate the interpretation of the violation of the BI by the experimental outcomes. Here, van Fraassen is a case in point. His interpretation of Bell-type experiments is that Bell-type experiments exhibit some phenomena which do not fit into one of our favorite models for scientific explanations: the common cause model. This interpretation is epistemological rather than ontological: it constraints the kinds of models that are legitimate, not the way the world is. ${ }^{47}$ We do not see how one could argue that an anti-realist like van Fraassen is committed, in investigating the violation of the BI, to the existence of the

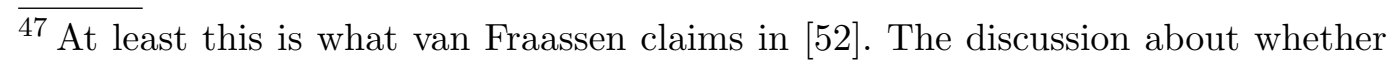
or not van Fraassen can stay at the level of models without drawing metaphysical conclusion is beyond the scope of this paper. 
joint probabilities in any ontologically robust sense.

From a more general point of view, we would like to stress that to accept a probabilistic physical theory does not involve any commitment to any particular interpretation of probabilities. ${ }^{48}$ Scientific realists as well as scientific empiricists can choose to interpret the probabilities involved in the theory as being:

(a) either objective or subjective;

(b) either reducible or irreducible to physical properties.

That some probabilities can be defined formally does not imply that they "exist" in any ontologically strong sense.

We maintain that the converse of this last statement holds as well: that some probabilities cannot be defined formally does not imply that they do not "exist", which brings us to the third component of (1), i.e. that to be realist about a theory that contains probabilities is sufficient for being committed to the "existence" of and only of the probabilities that are well defined within the theory. The point is easy to make in the case where one takes an empirical stance toward quantum mechanics. In this case, that some probabilities are not formally defined in the theory does not imply that they do not "exist", since a theory that pretends only to be empirically adequate does not preclude

\footnotetext{
$\overline{{ }^{48} \mathrm{CUT}}$ ? It is striking that the core of many arguments of the objectors to Fine up to now has been to vary the interpretations of the probabilities in Fine's results: Shimony and Redhead consider counterfactual interpretations, Svetlichny et al. consider a Church-von Mises interpretation. The reason for this, it seems to us, is that Fine's argument hinges on a specific interpretation of the probabilities.
} 
the "existence" of probabilities not contained in the formalism itself. But even for the realist, that some objects are not defined in a given formalism does not imply that these objects do not exist at the ontological level. For example, a defendant of a realist stance toward the Many-Worlds interpretation may believe that worlds exist as supervenient patterns emerging within the wave function, while explicitly avoiding to formally define them within the formalism. ${ }^{49}$ One important aim of the interpretational work on physical theories is precisely to 1 . distinguish clearly, within the formalism, what is physically significant from what is an artifact of the mathematical construction, and 2. complement the ontology with these entities which gives us a consistent view of a world in which the theory can be true.

The upshot is that, unless one accepts additional assumptions about realism and the interpretation of probabilities, the first step of Fine's last argument for his strong interpretation of his result does not stand better than the second. At the end, Fine's last line of argument seems to fail, unless further assumptions are made.

\section{Conclusion}

We have argued that:

- Fine's argument that the definition of joint probabilities is a hidden assumption in the traditional derivations of the BI holds only for a restricted class of h.v. theories, which were ruled out by previous theorems;

\footnotetext{
$\overline{49}$ This, in order to avoid the preferred basis problem. For more details on this, see [53], [54].
} 
- Fine's argument, to the effect that the existence of his Prism Models is a de facto argument against the traditional interpretation of Bell-type results, does not hold, because Prism Models are incompatible with some quantum statistical predictions;

- Fine's argument that the converse of his derivation shows that h.v. research programs start off with an inconsistent set of assumptions holds only under strong assumptions about the ontological status of probabilities.

It seems to us, then, that Fine fails to prove that his theorem has to be interpreted in a strong way, namely as undermining the traditional interpretation of Bell-type results in terms of local realism. Bell-type theorems and Bell-type results are still relevant for the interpretation of quantum theory and quantum phenomena.

That said, the framework of statistical variables, which Fine has been advocating as the appropriate one for quantum probabilities, is interesting, especially when fleshed out with a Bohmian interpretation. Further, Fine's Prism Models constitute a competitive model for the outcomes distributions to date, and should probably be given more attention.

\section{References}

[1] Araki, H. and Yanase, M. (1960). Measurement of Quantum Mechanical Operators, Physical Review, 120(2) 622-26.

[2] Aspect A. (1983). Trois tests expérimentaux des inégalités de Bell par mesure de corrélation de polarisation de photons. Thèse d'Etat, Orsay.

[3] Aspect, A. (2002). Bell's theorems: the naive view of an experimentalist, in R. 
A. Bertlmann and A. Zeilinger (Eds.), Quantum [UN]speakables - From Bell to Quantum Information, New York: Springer.

[4] Aspect, A., Dalibard, J. and Roger, G. (1982). Experimental test of Bell's inequalities using time-varying analysers, Physical Review Letters, 49, 1804-07.

[5] Bell, J.S. On the Einstein-Podolski-Rosen paradox, in [9], 14-21.

[6] Bell, J.S. On the problem of hidden variables in quantum mechanics, in [9], $1-13$.

[7] Bell, J.S. Introduction to the hidden-variable question, in [9], 29-39.

[8] Bell, J.S. The theory of local beables, in [9], 52-62.

[9] Bell, J.S. (1987). Speakable and Unspeakable in Quantum Mechanics, Cambridge: Cambridge University Press.

[10] Bell, J.S. (2004) (2nd edition). Speakable and Unspeakable in Quantum Mechanics, Cambridge: Cambridge University Press.

[11] Brown, H. R. (1991). Nonlocality in Quantum Mechanics, Part II, Proceedings of the Aristotelian Society, Supplementary Volume LXV: 141-159.

[12] Clauser, J.F. and Horne, M.A. (1969). A Proposed experiment to test local hidden-variable theories, Physical Review Letters, 23, 880-884.

[13] Clauser, J.F. and Horne, M.A. (1974). Experimental consequences of objective local theories, Physical Review D, 10, 526-35.

[14] Clifton R. and Pagonis, C. (1995). Unremarkable Contextualism: Dispositions in the Bohm Theory, Foundations of Physics, 25(2), 281-96.

[15] Cushing J.T. and McMullin E. (1989). Philosophical Consequences of Quantum Theory, Notre Dame: University of Notre Dame Press. 
[16] Dickson, M. (1996). Determinism and locality in quantum systems, Synthese, $107(1), 55-82$.

[17] Dowe, P. (2000). Physical Causation, by Cambridge: Cambridge University Press.

[18] Fine, A. (1968). Logic, probability,and quantum theory, Philosophy of Science, $35,101-11$

[19] Fine, A. (1971). Probability in Quantum Theories and in Other Statistical Theories, in Mario Bunge (Ed.), Problems in the Foundations of Physics (pp. 19-92), New York: Springer.

[20] Fine, A. (1973). Probabilities and the interpretation of quantum mechanics, British Journal for the Philosophy of Science, 24, 1-37.

[21] Fine, A. (1982a). Hidden variables, joint probability, and the Bell inequalities, Physics Review Letters, 48, 291-5.

[22] Fine, A. (1982b). Joint distributions, quantum correlations, and commuting observables, Journal of Mathematical Physics, 23, 1306-10.

[23] Fine, A. (1982c). Some local models for correlation experiment, Synthese, 50, 279-94.

[24] Fine, A. (1986). The Shaky Game, Einstein Realism and the Quantum Theory, Chicago: The University Chicago Press.

[25] Fine, A. (1989). Do correlations need to be explained?, in J. T. Cushing and E. McMullin (Eds.), Philosophical Consequences of Quantum Theory (pp. 175-94), Notre Dame: University of Notre Dame Press.

[26] Fine, A. (1989). Correlations and Efficiency: Testing the Bell inequalities, Foudations of Physics, 19:5, 453-477. 
[27] Fine, A. (1991). Inequalities for nonideal correlation experiments, Foundations of Physics, 21, 365-378.

[28] Fine, A. (1999). Locality and the Hardy theorem, in J. Butterfield and C. Pagonis, From physics to philosophy, Cambridge: Cambridge University Press.

[29] Gleason, A.M. (1957). Measures on closed Subspaces of a Hilbert Space, Journal of Mathematics and Mechanics, 6, 885-93.

[30] Howard, D. (1985). Einstein on Locality and Separability, Studies in History and Philosophy of Science, 16: 171-201.

[31] Jarrett, J.P. (1984). On the physical significance of the locality conditions in the Bell arguments, Nos 18, 569-589.

[32] Jarrett, J.P. (1989). Bells theorem: A guide to the implications, in [15], 61-79.

[33] Kochen S. and Specker E. (1967). The problem of hidden variables in quantum mechanics, Journal of Mathematics and Mechanics, 17, 59-87.

[34] Larsson, J-A (1999). Modeling the singlet state with local variables, Physics letters, A256, 245-252.

[35] Larsson J-A. and Gill R. D. (2004). Bell's inequality and the coincidence-time loophole, Europhysics letters, 67, 707-13.

[36] Maudlin, T. (1994). Quantum Non-Locality and Relativity, Oxford U.K. and Cambridge MA: Blackwell.

[37] Mermin, N.D. (1986). Generalizations of Bell's Theorem to higher spins and higher correlations, in L.M. Roth and A. Inomato, Fundamental Questions in Quantum Mechanics (pp.7-20), London: Gordon and Breach.

[38] Price, H. (1996). Time's Arrow and Archimedes' Point: New Directions for the Physics of Time, New York: Oxford University Press, 1996. 
[39] Redhead, M. (1987). Incompleteness, Nonlocality, and Realism, A Prolegomenon to the Philosophy of Quantum Mechanics, Gloucestershire: Clarendon.

[40] Redhead, M. (1991). Nonlocality in Quantum Mechanics, Part I, Proceedings of the Aristotelian Society, Supplementary Volume LXV: 119-140.

[41] Shimony, A. (1984). Contextual hidden variables theories and the Bell's inequalities, British Journal for the Philosophy of Science, 35(1), 25-45.

[42] Shimony, A. (1986). Events and processes in the quantum world, in R. Penrose and C. Isham (eds), Quantum Concepts in Space and Time, Oxford: Oxford University Press, 182-203.

[43] Shimony, A. (Summer 2005). Bell's theorem, in E. N. Zalta (Ed.), The Stanford Encyclopedia of Philosophy, http://plato.stanford.edu/ archives/spr2005/entries/physics-Rpcc/

[44] Suppes, P. and Zanotti, M. (1976). Necessary and sufficient conditions for existence of a unique measure strictly agreeing with a qualitative probability ordering, Journal of Philosophical Logic, 5, 431-438.

[45] Svetlichny G., Redhead M.,Brown H. and Butterfield J. (1988). Do the Bell inequalities require the existence of joint probability distributions?, Philosophy of Science, 55, 387-401.

[46] Szabó, L. E. (2000). On Fine's resolution of the EPR-Bell's problem, Foundations of Physics, 30, 1891-1909.

[47] Szabó, L. E. (2007). The Einstein-Podolsky-Rosen Argument and the Bell Inequalities, in J. Fieser and B. Dowden, The Internet Encycopedia of Philosophy, http://www.iep.utm.edu/e/epr.htm.

[48] Szabó L. E. and Fine A. (2002). A local hidden variable theory for the GHZ experiment. Physics Letters, A295, 229-240. 
[49] Teller, P. (1979). Quantum Mechanics and the Nature of Continuous Physical Quantities, Journal of Philosophy, 76(7), 345-62.

[50] Teller P. (1984). The Projection Postulate of Quantum Mechanics: A New Perspective, Philosophy of Science, 51(3), 369-95.

[51] Teller P. (1989). Relational Holism and the Bell's inequality, in [15], 208-18.

[52] Van Fraassen, B. C. (1989). The charybdis of realism: epistemological implications of Bells inequality, in [15], 97-113.

[53] Wallace, D. (2002). Worlds in the Everett interpretation, Studies In History and Philosophy of Modern Physics, 33 (4), 637-661.

[54] Wallace, D. (2003). Everett and structure, Studies In History and Philosophy of Modern Physics, 34 (1), 87-105.

[55] Weihs G., Jennewein T., Simon Ch. ,Weinfurter H. and Zeilinger A., Violation of Bell's inequality under strict Einstein locality conditions, Physics Review Letters, 81, 5039-43.

[56] Zeilinger A., Experiment and the foundations of quantum physics, Review of Modern Physics, 71, S299-97. 